\title{
La escuela, un espacio social fundamental para la democratización peruana. Un estudio comparado entre la propuesta educativa tradicional y una propuesta educativa alternativa
}

\section{Lars Stojnic Chávez}

\author{
Pontificia Universidad Católica del Perú y \\ Consorcio de Investigación Económica y Social
}

Lars Stojnic es Licenciado en Sociología de la PUCP. Actualmente es Oficial de Proyectos en el Consorcio de Investigación Económica y Social y desde el 2006 es docente contratado en la PUCP. Ha trabajado en proyectos referidos a descentralización, democracia y participación y gestión escolar, destacando su participación en los programas de educación de DFID, la GTZ y en el Colegio La Casa de Cartón.

Este artículo ha sido elaborado a partir de la investigación del mismo nombre, presentada por el autor en agosto del 2006 como tesis de sustentación de licenciatura en Sociología de la Pontificia Universidad Católica del Perú (PUCP) y que fue ganadora del concurso de Tesis de Licenciatura organizado por la SIEP en el marco del Primer Seminario de Investigación Educativa en noviembre del 2008.

Agradezco a Patricia Ruiz Bravo, a Fanny Muñoz, a las dos instituciones en las que se llevó a cabo la investigación, a los estudiantes por todo el tiempo que me brindaron y por la sinceridad y apertura de sus opiniones e intervenciones, a Ricardo Cuenca, a mi familia por su apoyo incondicional y a mi Clau por regalarme magia cada día. 


\title{
La escuela, un espacio social fundamental para la democratización peruana. Un estudio comparado entre la propuesta educativa tradicional y una propuesta educativa alternativa
}

\begin{abstract}
Resumen
La investigación enfocó sus esfuerzos en analizar cómo es que la escuela asume el rol de formación y socialización de individuos democráticos, no sólo con conocimientos teóricos, sino como sujetos capaces de reproducir en su vida cotidiana y en su relación con sus semejantes y su medio social, discursos y prácticas sociales de tipo democrático. Se eligió la escuela como objeto de estudio porque es la única institución formalmente reconocida con el objetivo de formar a las generaciones futuras y porque se presenta como el primer espacio en que las personas se relacionan formalmente con lo público. El estudio tuvo una metodología de caso de estudio de dos propuestas educativas distintas, una que denominamos "tradicional" y otra "alternativa", para analizar si existían diferencias en la formación democrática de sus estudiantes. La corriente a la que se adscribe la investigación ${ }^{1}$, plantea que la democracia se funda en la libertad de los individuos, la conformación de una comunidad política y la formación ciudadana. Partiendo de ese enfoque se identificaron tres cualidades para evaluar los logros de cada institución en el proceso de formación democrática: el compromiso social, la participación pública y el manejo de la normatividad de sus estudiantes. Los resultados mostraron, por un lado, disposiciones distintas de los estudiantes de ambas instituciones en relación a las dimensiones de estudio y por otro lado, que la propuesta alternativa analizada facilitaba el desarrollo de mayor disposición democrática de sus estudiantes, en comparación a la tradicional.
\end{abstract}

Palabras clave: Democracia, Educación, Ciudadanía, Socialización, Escuela.

1 Tomado del trabajo de Chantall Mouffe (1999), "El retorno de lo político". 


\title{
The school, a key social space for peruvian democratization. A study comparing the traditional educative proposal and an alternative educative proposal
}

\begin{abstract}
Summary
The investigation focused its efforts in analyzing how is it that school assumes the roles of formation and socialization of democratic individuals, not only with theoretical knowledge, but also as subjects capable of reproducing social discourses and practices of the democratic kind in their everyday life and in their relationship to their peers and their social environment. The school was chosen as the object of study, firstly because it's the only recognized institution with the goal of forming future generations; and secondly because it presents itself as the first space in which people formally relate to all that is public. The study had a methodology of case study of two distinct educative proposals, one that we denominate "traditional" and another one known as "alternative" to analyze if there were differences in the democratic formation of students. Theoretically the research was based on a thinking current ${ }^{2}$, which states that democracy is founded on individuals' freedom, the formation of a political community and citizen's formation. Starting on this perspective, three democratic qualities were identified to evaluate the achievements of each school in the process of democratic formation: social commitment, public participation and individual normativity management. The results showed, on one side, the attitude differences in students from both schools in relation the dimensions of the study and on the other hand, presented evidence that the analyzed alternative proposal is facilitating in a greater measure the development of democratic disposition in their students, compare to the other proposal.
\end{abstract}

Key words: Democracy, Education, Citizenship, Socialization, School.

2 Taken from Chantall Mouffe's work (1999), "The return of the political". 
“...sólo se podrá considerar que una sociedad es democrática si es que las instituciones que dan sustento a la convivencia social, son reflejo cotidiano de valores y prácticas democráticas."

Alain Touraine

\section{Introducción}

A finales del 2000 y luego de 10 años en que el Perú vivió sumido en un régimen dictatorial ${ }^{3}$, las elites políticas y gubernamentales mantuvieron un discurso interesado en la consolidación del proceso de "democratización". Éste, consideramos, se enfocó principalmente en asegurar aspectos formales del funcionamiento del sistema político y de la relación entre el Estado y los individuos.

Sin dejar de reconocer la importancia de estos elementos, es importante recordar que cualquier sistema social y/o político existe sólo si se reproduce por la interacción cotidiana de los sujetos y las instituciones, por lo que cabría preguntarnos si nuestra cotidianeidad social y política es reflejo hoy de una convivencia democrática. Esta reflexión llevó a dos cuestionamientos que fueron la base de la investigación, sobre la cual se ha elaborado el presente artículo: ¿asegurar los aspectos formales son indicadores suficientes para determinar que nuestra sociedad es democrática? y ¿de qué manera se viene fortaleciendo nuestra cotidianeidad para ser reflejo de una convivencia democrática?

Giroux (1993, p. 14) señala que las instituciones educativas “. . . figuran entre los pocos espacios de vida pública en los que los estudiantes, jóvenes o adultos, pueden experimentar y aprender el lenguaje de la comunidad y de la vida pública democrática". Coincidiendo con dicho planteamiento, se eligió a la institución educativa (II.EE.) como objeto de estudio, debido al rol que debería asumir para la consolidación de la democracia, a través de la formación de ciudadanos democráticos. En tal sentido, esperamos que el artículo promueva la reflexión sobre el rol de dichas instituciones en nuestro proceso de democratización.

El artículo está dividido en seis secciones: la primera, describe los enfoques conceptuales y presenta una caracterización de las propuestas educativas que denominamos tradicional y alternativa; la segunda, presenta el objetivo 
y metodología que sustentaron la investigación; la tercera sección, plantea los principales hallazgos de la investigación, cerrando el artículo con algunas reflexiones finales.

\section{Aproximaciones conceptuales}

La investigación analizó el rol que cumplen las instituciones educativas en el proceso de democratización de la sociedad peruana, por lo cual, se delimitaron dos conceptos básicos: en primer lugar, la idea de democracia y su relación con la construcción política denominada "ciudadanía"; y en segundo lugar, se conceptualizó a la II.EE. como una institución política.

En relación al concepto de democracia, nos adscribimos a una corriente de pensamiento 4 que recupera y combina los aspectos centrales del liberalismo y el comunitarismo. La "democracia liberal", por un lado, se funda en los ideales de igualdad y libertad de los individuos frente al Estado y a la sociedad. Siguiendo esta perspectiva, el fin del Estado sería plantear un marco jurídico que permita que los individuos alcancen sus objetivos (Bobbio, 1989) y que no permita que el Estado adopte características autoritarias (Gamio, 1999)5. Por otro lado, la democracia desde una perspectiva comunitarista sostiene que la esencia política de las personas sólo se concreta cuando éstos se involucran en la comunidad a la que pertenecen en búsqueda de alcanzar el bien común (Gamio, 1999) ${ }^{6}$.

La tercera corriente, plantea que la democracia se sustenta de igual forma, en la protección de las libertades y derechos de los individuos, como en el fortalecimiento del compromiso de éstos ante su comunidad. Plantea como pilares de la democracia la libertad de los individuos, el fortalecimiento de una comunidad política y la formación ciudadana. Coincidimos con esta corriente en que la institucionalización de la democracia exigirá:

4 Desarrollado en el trabajo de Chantall Mouffe (1999), "El retorno de lo político".

5 Robert Dahl destaca el derecho al voto como decisivo en la construcción de la democracia desde una perspectiva liberal, ya que permite que los sujetos se involucren en los procesos políticos (Benavont, 1996).

6 El autor hace referencia por ejemplo a que Tocqueville (1984), en "La democracia en América", sostiene que uno de los efectos perniciosos de algunas democracias modernas es que los individuos, a cambio de cierta seguridad y prosperidad que asegura el Estado, se retraen a sus vidas privadas sin ejercer sus derechos políticos. 
- La implementación de un conjunto de instituciones cuyos objetivos sean, la protección y promoción de libertades y derechos individuales y colectivos, así como el ordenamiento de la convivencia social.

- El fortalecimiento de una cultura democrática cotidiana, que se refleje en las actitudes y relaciones de sujetos que se conciban como ciudadanos pertenecientes y responsables de su comunidad.

En sus planteamientos, esta corriente destaca al ciudadano como agente de cambio social, señalando que la ciudadanía, es una categoría política que los sujetos desarrollamos o no, a través de nuestra relación permanente con las instituciones y con nuestros semejantes, como parte de una comunidad mayor. En tal sentido, aunque la definición de la ciudadanía debe incluir los derechos y libertades jurídicas, también debe considerar la incorporación de la idea del bien común y de la responsabilidades cívicas, políticas y sociales de los individuos (Mouffe, 1999). El concepto de ciudadanía remite a la apropiación de las personas de una serie de derechos y libertades, pero también de un sentido de compromiso hacia lo público, entendiéndolo como el espacio en el que se define la convivencia social y política.

Como señala Cotler (2001), una condición indispensable y probablemente la más difícil de enfrentar es la formación de una cultura que sustente la consolidación de la democracia en el Perú. Es en dicho proceso que planteamos que la II.EE. tiene un papel predominante, desde su dimensión política, en la formación de personas que se apropien de la categoría de ciudadanía.

Con respecto al segundo concepto, entender el sentido político de las II.EE. parte de comprender su rol como medio de socialización, entendiendo dicho proceso como el que facilita que niñas y niños adopten conciencia de sí mismos e incorporen conocimientos y prácticas de la sociedad en la que han nacido (Giddens, 2002). El sujeto sería tal porque incorpora las instituciones de su comunidad a su propia conducta ${ }^{7}$ y aunque existen diversos medios de socialización, consideramos que la II.EE es uno de los más importantes en la formación democrática, debido a dos hechos principalmente.

En primer lugar, es la única institución social formalmente reconocida con el objetivo de formar a las generaciones futuras. Bordieu (1997) señala que a

7 Idea del "otro generalizado" desarrollada por George Mead (1972) en su texto Espíritu, persona y sociedad. Parte III. 
la institución escolar se le atribuye un rol esencial en la formación de los individuos como "criaturas del Estado". Éste, por ejemplo, define los aprendizajes que se espera que los estudiantes incorporen y de tal manera, aprovecha el rol de las II.EE. en el proceso de formación de las mentes, valores y prácticas de los individuos.

En segundo lugar la institución educativa es el primer espacio en que las personas se relacionan formalmente con lo público. Es ahí que tenemos la oportunidad, por primera vez, de enfrentar un ambiente marcado por reglas formales, por figuras de autoridad y en que nos relacionamos con nuestros semejantes dentro de marcos institucionales. Así, los estudiantes no sólo aprenden de los conocimientos que se trasmiten en las aulas, sino que además se forman a través de las relaciones con y dentro de la institución que los alberga.

Históricamente, la educación ha sido concebida como un espacio social de formación en valores y actitudes según el norte socialmente definido ${ }^{8}$; y aunque en la actualidad se priorice su dimensión académica, "la educación, como acción de influir en las personas, es profundamente ética y política por propia naturaleza" (Apple, 1996, p. 57). La cotidianeidad escolar influye en la manera que los estudiantes aprenden a vivir en sociedad y en tal sentido, es importante reflexionar sobre sus características actualmente. Por tal razón, presentamos los principales hallazgos de la revisión de estudios que abordaban la cotidianeidad escolar peruana.

Ésta fue valiosa para identificar algunas características de las II.EE. públicas, las cuáles consideramos que podrían incidir en la formación democrática de sus estudiantes. Una característica general recogida fue que en las últimas décadas las instituciones educativas han asumido la transmisión de conocimientos como su rol principal, razón por la cual, la democracia vendría siendo abordada como un contenido y sin ningún correlato práctico y vivencial. Además, las II.EE. vendrían reproduciendo formas de organización y relaciones contrarias a una convivencia democrática, como lo muestran los aspectos ${ }^{9}$

8 “'Los ciudadanos de un Estado deben ser educados siempre en consonancia con la Constitución de su Estado', argumentó Aristóteles. 'Las leyes de la educación deben ser relativas a los principios de gobierno' señaló Montesquieu, como lo hicieron Durkheim y muchos teóricos sociales contemporáneos" (Guttman, 2001).

9 En la investigación que sirve de base para el presente artículo se identificaron cinco características, aunque por cuestiones de espacio, en este caso sólo presentaremos tres de ellas. Las dos restantes, eran la exaltación del disciplinamiento de los estudiantes, caracterizado por un orden semi militarizado y la discriminación que sufren los estudiantes dentro de la escuela y que se reforzaría por la pasividad de los docentes y autoridades responsables de la escuela. 
sistematizados y que sirvieron de referencia para la metodología de la investigación.

En primer lugar, en las II.EE. se reproduciría una perniciosa "anonimación" de los estudiantes. Autores como León y Staeheli (2001), Iguiñis y Dueñas (1998) plantean que un grave problema del espacio escolar es el desinterés de las autoridades educativas por reconocer y valorar la individualidad de los estudiantes. Dicha anonimación dificultaría el crecimiento personal de los estudiantes, al limitar el desarrollo de su autoestima.

Un segundo rasgo identificado, es que el único espacio dentro de las II.EE. en el que los estudiantes encontrarían algún tipo de reconocimiento sobre su capacidad de agencia es el aula y sobre cuestiones académicas. El rol principal que se les asigna a los estudiantes es la adquisición de conocimientos y de ciertos modos de comportarse que la sociedad les "demanda"10 (León y Staeheli, 2001), por lo que no se les reconocería un rol en los espacios públicos escolares. Así mismo, la inexistencia y/o ineficacia de espacios de participación para los estudiantes, como lo que sucede con los municipios escolares (Carbajo y Serna, 1999), provocaría su desinterés y desmotivación antes temas públicos o institucionales.

Finalmente, las investigaciones revisadas muestran que los estudiantes no participan en la definición de las normas escolares y éstas les son impuestas sin que interese su opinión. Un claro ejemplo, como Ames (1999) señala, es que aún en la definición de normas del aula, el docente las dicta, siendo el único rol de los estudiantes reafirmarlas, sin posibilidad de crítica u oposición. El que no se incluya a los estudiantes como actores legítimos del proceso de definición de normas institucionales, evidenciaría el poco reconocimiento de éstos como actores legítimos en el espacio público escolar.

Estas características dan cuenta de las situaciones a la que se enfrenta un porcentaje amplio de estudiantes de instituciones públicas. Ahora bien, aunque sólo un par de estudios abordaban la problemática de las II.EE. particulares, consideramos que éstas, a pesar de facilitar una mejor preparación académica y de su mejor acceso a recursos, en muchos casos reproducirían situaciones muy parecidas a lo descrito. Así pues, nos referiremos a instituciones con propuestas educativas tradicionales ${ }^{11}$ en aquellos casos en que institucionalmente se reproduzca una fuerte jerarquización, con patrones impositivos de autoridad y con relaciones de discriminación y exclusión.

10 Como la capacidad de mantenerse en silencio y acatar lo que los adultos señalan.

11 Independientemente de si dichas instituciones son públicas o privadas. 
En contraposición, y desde la revisión de los pocos textos que abordan dicha problemática (Benavides, Villarán y Cueto(1999); Kallinowski, Ruiz y Dueñas (1996); y Moragues (1996))., las instituciones con propuestas educativas "alternativas" serían aquellas, públicas o privadas, que enfatizarían la construcción de un espacio educativo que promueva condiciones de convivencia constructiva, que busquen incluir a sus estudiantes en los asuntos públicos y que fomenten su involucramiento frente al cambio social.

Así mismo, lo desarrollado permitió identificar algunas cualidades que consideramos claves para analizar la disposición de las personas frente a la democracia. Se eligieron tres como las dimensiones de estudio de la investigación ${ }^{12}$, sin embargo, en el presente artículo sólo analizaremos los resultados en relación a dos de ellas ${ }^{13}$.

- Compromiso social, entendida como la capacidad de cada persona de identificarse con la comunidad a la que pertenecen y asumir responsabilidad con su desarrollo y;

- Participación pública, entendida como el involucramiento de los sujetos, de forma permanente y activa, en los asuntos de interés colectivo.

Se eligió concentrar la investigación en estas cualidades, en primer lugar, porque abordan los dos aspectos centrales del enfoque de democracia al que nos adscribimos ${ }^{14}$. En segundo lugar, porque dan cuenta de cuatro de las características que se sistematizaron y quisimos analizar su influencia en la formación democrática. Finalmente, porque el análisis de dichas cualidades podía aproximarnos a comprender el vínculo y la incorporación de los estudiantes al espacio público escolar.

12 Reconocemos que en dicho proceso, hay otras cualidades ciudadanas como la tolerancia, la autonomía, el reconocimiento de las diferencias, sentido de pertenencia, etc., que también sería fundamental promover e investigar; sin embargo, se optó sólo por tres, debido a los recursos limitados con los que se contaba para la investigación.

13 La tercera cualidad era "manejo de la normatividad", entendido como el nivel de apropiación y respeto de los sujetos hacia las normas y estructuras que enmarcan la convivencia cotidiana.

14 La consolidación de una institucionalidad que enmarque la cotidianeidad y la formación de ciudadanos responsables de su comunidad. 


\section{Objetivos y metodología de la investigación}

La investigación abordó el proceso de democratización del Perú en referencia al fortalecimiento de una cultura democrática, entendiendo que ésta se reproducirá sólo si los sujetos se apropian cotidianamente de la categoría de "ciudadanía".

Coincidimos con Touraine (1995) en que la aproximación al proceso de democratización debe realizarse desde las distintas instituciones y organizaciones que enmarcan la vida social. Por tal razón, el objetivo de la investigación fue analizar la influencia de la propuesta educativa de las II.EE. ${ }^{15}$-reflejada en su cotidianeidad institucional- en el desarrollo de la disposición democrática de sus estudiantes en relación con lo público.

La investigación se llevó a cabo en dos II.EE privadas limeñas y de educación mixta. Los objetivos específicos de la investigación fueron identificar la disposición cotidiana de los estudiantes hacia la democracia y analizar la influencia de la cotidianeidad institucional escolar ${ }^{16}$ en tal sentido. La hipótesis principal fue que para que la institución educativa se constituya en agente promotor de una cultura democrática es indispensable que su cotidianeidad institucional sea reflejo de una propuesta educativa democrática.

La metodología fue de tipo cualitativo y partió del estudio de caso de las dos instituciones antes mencionadas. Consideramos que utilizar el caso de estudio era una alternativa pertinente para conocer con mayor profundidad la cotidianeidad de ambas instituciones, así como la influencia de las mismas en las disposiciones de sus estudiantes.

Se eligieron ambas instituciones, en primer lugar, porque, aunque sería impreciso indicar que en los discursos institucionales de cada una sólo se encontraron rasgos de una de las dos propuestas, sí se consideró que una de ellas presentaba más características de una propuesta educativa tradicional, mientras que la otra, más de una propuesta alternativa. Una segunda razón fue la facilidad del acceso geográfico a las dos.

En tercer lugar, se eligieron debido a que sus estudiantes provenían de una condición socio-económica similar; la pensión aproximada de ambas escuelas

15 Aunque el estudio se concentra en la II.EE, reconocemos que existen otras instituciones sociales que cumplen un rol importante en la consecución de un propósito tan complejo como el consolidar una cultura democrática, como las familias y los medios de comunicación por ejemplo.

16 Incluía el análisis de la organización y estructura de poder escolares, los recursos pedagógicos utilizados y las relaciones sociales que se reproducían en cada institución. 
(sin tomar en cuenta estudiantes becados) era de 500 soles y la residencia de los estudiantes se concentraba en distritos de ingreso medio ${ }^{17}$. Finalmente, debido a que en ambas instituciones el número de estudiantes por salón era de máximo 25 estudiantes; en la institución tradicional cada grado poseía dos secciones y en la alternativa sólo una.

El estudio se centró en estudiantes de cuarto y quinto de secundaria porque al estar en los últimos grados de secundaria, consideramos que serían un grupo representativo de la formación escolar recibida y con mayor capacidad crítica y reflexiva sobre la cotidianeidad de sus instituciones ${ }^{18}$.

El proceso de recojo de información se abordó en dos etapas y aprovechando distintos instrumentos y procedimientos que explicamos a continuación; éstos pueden encontrarse en los anexos de la investigación que sirvió de base al artículo (Stojnic, 2006). La primera, tuvo como objetivo aproximarnos a conocer si es que los estudiantes presentaban disposiciones diferenciadas según la institución de la que procedían, en relación a las cualidades democráticas elegidas. El recojo de información se hizo, en primer lugar, a través de una encuesta que llenaron los estudiantes de ambos grados que asistieron el día de aplicación del instrumento.

En total la encuesta fue llenada por 124 estudiantes, 36 de la institución alternativa y 88 estudiantes de la II.EE. tradicional ${ }^{19}$. El instrumento permitió recoger información acerca de ambos grupos, los que presentaron características similares:

- $\quad$ El 54.5\% de los encuestados de la institución tradicional eran de cuarto de secundaria, mientras que $45.5 \%$ eran de quinto. En el caso de la II.EE alternativa, $47.2 \%$ de los encuestados eran de cuarto y $52.8 \%$ de quinto de secundaria.

- El $81.8 \%$ de los estudiantes de la propuesta tradicional eran del sexo masculino, al igual que el 55.6\% de la institución alternativa. Del sexo femenino, eran el $18.2 \%$ de los encuestados de la II.EE. alternativa y el $44.4 \%$ de la tradicional.

17 Como Miraflores, Barranco, San Miguel, Comas, Independencia, Jesús María, Pueblo Libre, Chorrillos, etc.

18 Se pudo haber tomado otras variables en consideración, como tiempo que los estudiantes estudiaban en la institución o características de la familia de procedencia, sin embargo, éstas no se tomaron en cuenta por las limitaciones de recursos.

19 La diferencia en la cantidad de encuestados se debió, como se explica líneas arriba, a que una escuela contaba con una sección por grado, mientras que la segunda con dos. 
- El $86 \%$ de los encuestados de la II.EE. tradicional y el $73 \%$ de la alternativa tenían entre 15 y 16 años de edad.

- El $89.9 \%$ de los estudiantes de la institución tradicional y $91.7 \%$ de la II.EE. alternativa señalaron que su situación socio económica era de clase media.

- El $82.7 \%$ de los estudiantes de la institución tradicional señaló que sus padres tenían un nivel educativo superior universitario, mientras que el $74 \%$ indicó lo mismo con respecto a sus madres. De la II.EE. alternativa, $77.1 \%$ señaló que sus padres tenían un nivel educativo superior universitario y el $68.6 \%$ indicó lo mismo sobre sus madres.

La encuesta contaba con 50 preguntas y contenía, por un lado, preguntas que buscaban que los estudiantes opinasen, desde una posición del "deber ser", sobre su rol en la escuela, sus percepciones sobre la democracia, temas de coyuntura, etc. Por otro lado, se plantearon preguntas que enfrentaban al estudiante con casos cotidianos, sobre los cuáles se solicitaba que indicaran sus posibles reacciones. Se aprovecharon ambos tipos de preguntas con el afán de evidenciar la coherencia entre los discursos y las posibles reacciones de los estudiantes.

En segundo lugar, se llevó a cabo un primer grupo focal con nueve estudiantes de la II.EE. tradicional y once de la alternativa, que hubiesen completado la encuesta. En el caso de la primera, los participantes fueron 6 mujeres y 3 hombres y ocho de ellos eran de quinto de secundaria; en el caso de la institución alternativa, 8 estudiantes eran mujeres y 3 eran hombres, mientras que 6 de los 11 cursaban quinto de secundaria. Ambos grupos focales duraron aproximadamente $1 \mathrm{hr}$. $30 \mathrm{~min}$. y las preguntas estuvieron dirigidas a conocer cuáles eran sus concepciones sobre el rol que tenían en la institución, qué tan responsables se sentían en relación al espacio escolar y sobre su percepción y manejo de las normas escolares.

La segunda etapa de la investigación, estuvo dirigida a identificar los factores de la institucionalidad escolar que pudiesen influir en las disposiciones que los estudiantes presentaron. Los procedimientos utilizados fueron, en primer lugar, el análisis de las estructuras de organización y de decisión de ambas instituciones, con el propósito de conocer las estructuras de poder, las jerarquías institucionales y las posibilidades de participación de los estudiantes.

En segundo lugar, se llevó a cabo observaciones de aula y de otras acti134 I vidades escolares desde el inicio del día escolar, de tipo no participante. En la 
institución de propuesta tradicional se realizaron cuatro sesiones de 4 horas cada una, en la se acompañó a los dos grados de 4ํ y 5으 de secundaria (una sesión con cada grado). En la institución alternativa fueron tres sesiones de 4 hrs. y 30 min. cada una, en las que se acompañó un día a 4ํ de secundaria y en dos sesiones a 50 de secundaria.

Durante las observaciones realizadas se buscó identificar los estilos de autoridad que institucionalmente se manejaban, el tipo de relaciones entre los actores escolares, el nivel de participación de los estudiantes y su manejo de la normatividad institucional. En cada sesión, aprovechando una guía de registro elaborada a partir de los resultados de la encuesta, se registraron aquellas situaciones que daban cuenta de las dimensiones señaladas; se prestó atención tanto a momentos institucionales, como el dictado de clase y actividades colectivas, así como los momentos considerados libres, como los cambios de hora, recreos y recesos.

En tercer lugar, se llevó a cabo una segunda ronda de grupos focales, en la que participaron 7 estudiantes de la II.EE tradicional ( 5 mujeres y 2 hombres $)^{20}$ y 9 de la alternativa ( 6 mujeres y 3 hombres) ${ }^{21}$, que hubiesen participado del primer grupo focal. Su propósito fue recoger las opiniones de los estudiantes sobre las estructuras de poder, formas de organización y convivencia cotidiana de sus instituciones. Finalmente, se realizaron entrevistas con los directores, con el propósito de recoger sus percepciones sobre la organización institucional, el rol de los estudiantes y las prioridades y estrategias aprovechadas para la formación de éstos.

La metodología aplicada fue lo más exhaustiva posible, dentro de lo posible, debido al interés por contar con distintas fuentes que permitieran corroborar la información levantada. Sin embargo, reconocemos algunos vacíos que podrían ser resueltos y mejorados en el futuro. Por un lado, que al abordar tres cualidades distintas, los instrumentos no lograron necesariamente abarcar todos los aspectos relacionados a cada una; por otro lado, habría sido útil la elaboración de escalas o índices de análisis a partir de la encuesta; y finalmente, hubiese sido más enriquecedor analizar los procesos escolares tanto en primaria como en secundaria.

20 Sólo uno de los participantes era de 4으 de secundaria.

214 de los 9 participantes cursaban 4을 de secundaria. 


\section{Principales hallazgos}

A continuación se presentan dos secciones con los principales hallazgos de la investigación en relación a las cualidades democráticas de compromiso social y participación pública. La primera sección presenta la disposición de los estudiantes de ambas instituciones frente a las dos cualidades ${ }^{22}$ y la segunda, plantea el análisis de la influencia de las propuestas educativas en el desarrollo de dichas disposiciones.

Es importante recordar que los resultados hacen referencia a dos casos de estudio y aunque consideramos que cada institución presenta rasgos que pueden llevar a que se les asocie con una de las dos propuestas, no es posible generalizar a partir de ellos. Reconocemos que al no haber podido controlar algunas variables -como el ambiente familiar, sus momentos de vida o la influencia de otros medios de socialización- no es posible atribuir lo encontrado directa y significativamente a las propuestas educativas. Sin embargo, consideramos que los resultados permiten una aproximación interesante a la cotidianeidad escolar de ambas propuestas y su posible influencia en la formación democrática de sus estudiantes ${ }^{23}$.

22 Ambas dimensiones se presentan de manera conjunta debido a la interrelación e influencia que consideramos que cada una de ellas tiene sobre la otra.

23 En la tercera dimensión analizada, con respecto al manejo de los estudiantes de las normas y acuerdos institucionales, no se encontraron mayores diferencias entre la disposición de ambos grupos de estudiantes. Los resultados mostraron que aunque más del $70 \%$ de los estudiantes encuestados señalaron que en ningún caso era justificable evadir las leyes, ante situaciones cotidianas, la gran mayoría señaló que adoptarían actitudes que contradicen las normas de convivencia y las normas legales. Sin embargo, los resultados nos mostraron que las razones que influían en el desarrollo de la misma actitud en los dos grupos de estudiantes eran distintas. En el caso de la II.EE. alternativa, un aspecto que influía en la relativización de las normas por parte de los estudiantes, era la falta de homogeneidad institucional por parte del cuerpo docente para presentar una autoridad firme que promoviese e hiciese respetar los acuerdos y reglas en la escuela. Otro aspecto, era que a medida que se avanzaba en los grados, el acompañamiento de los tutores y docentes en el refuerzo de acuerdos y normas de convivencia disminuía, debido a que se les consideraba "más grandes". En el caso de la II.EE. tradicional, un aspecto de influencia era que los estudiantes percibían la normatividad escolar como ajena, el cumplimiento de las mismas la incorporaba como una obligación y "escapar" de ellas como un logro. Dos aspectos importantes en este sentido eran que los estudiantes no participaban de la elaboración de las normas y que las autoridades escolares no demostraban la intención de hacer entender a los estudiantes el sentido de las mismas. 


\section{Compromiso social y participación pública de los estudiantes en su espacio escolar ${ }^{24}$}

Un primer hallazgo fue que la mayoría de estudiantes de ambas instituciones priorizaron aspectos relacionados al compromiso y a las posibilidades de participación de los ciudadanos en los asuntos públicos de la comunidad, como elementos claves en el fortalecimiento de la democracia.

Ante la pregunta "¿Qué es necesario para que una sociedad sea democrática?", 58.6\% de los estudiantes encuestados en la II.EE. tradicional y $69.4 \%$ de la alternativa, señalaron que era más importante asegurar espacios y mecanismos de participación, antes incluso que asegurar ciertas libertades y derechos individuales ${ }^{25}$. Cuando se les preguntó “¿Qué se necesita para que el Perú sea democrático?", $72 \%$ de los estudiantes de la institución alternativa y $58 \%$ de la tradicional, señalaron que se necesitaba principalmente la participación del pueblo y que éste ejercite sus derechos y responsabilidades.

Al cruzar dicha información con las percepciones de los estudiantes en relación a quién dependía el desarrollo de la II.EE., encontramos que más del $60 \%$ consideraba que ellos debían asumir un rol importante.

\section{TABLA 1}

¿De quién depende el desarrollo de la escuela?

\begin{tabular}{lccc}
\hline Institución & Director y/o docentes & $\begin{array}{c}\text { De alumnos igual } \\
\text { que directores y docentes }\end{array}$ & Total \\
\hline Tradicional & 29 & $\mathbf{5 9}$ & $88^{*}$ \\
& $33,0 \%$ & $\mathbf{6 7 , 0 \%}$ & $100 \%$ \\
Alternativo & 14 & $\mathbf{2 2}$ & 36 \\
$*$ & $38,9 \%$ & $\mathbf{6 1 , 1 \%}$ & $100 \%$ \\
\hline $\begin{array}{l}\text { Es importante señalar, que el total de encuestados hace referencia al total de los estudiantes que respondieron a } \\
\text { cada pregunta. Esto es importante porque pueden hallarse diferencias en el total para cada una de las preguntas } \\
\text { que se presenten. }\end{array}$
\end{tabular}

Los datos evidenciaban que en sus discursos los estudiantes no presentaban mayores diferencias, sin embargo, cuando se les preguntó “¿Cuál es tu

24 En la investigación se buscó abordar las cualidades desde una perspectiva más integral, por lo que se midió la disposición de los estudiantes no sólo frente al espacio escolar, sino además en su cotidianeidad fuera de la escuela. Sin embargo, en el artículo se enfoca el análisis de las cualidades sólo en relación al espacio escolar. 
prioridad como estudiante?", presentándole opciones referidas a su desarrollo individual, se encontraron diferencias según la institución de procedencia:

\section{TABLA 2}

La prioridad de los encuestados en su rol de estudiantes

\begin{tabular}{cccc}
\hline \multirow{2}{*}{ Institución } & $\begin{array}{c}\text { Sacar buenas notas/ } \\
\text { aprender y ser buen amigo }\end{array}$ & $\begin{array}{c}\text { Aportar para que } \\
\text { la escuela mejore }\end{array}$ & Total \\
\hline \multirow{2}{*}{ Tradicional } & $\mathbf{4 1}$ & 43 & 84 \\
& $\mathbf{4 8 , 8 \%}$ & $51,2 \%$ & $100 \%$ \\
Alternativa & 9 & $\mathbf{2 7}$ & 36 \\
& $25 \%$ & $\mathbf{7 5 \%}$ & $100 \%$ \\
\hline
\end{tabular}

Aunque, en ambos casos la mayoría de estudiantes eligió la opción "aprender y aportar para que su escuela mejore", los datos muestran que ante un caso cotidiano, casi la mitad de estudiantes de la institución tradicional optaron por anteponer su individualidad antes que al colectivo. Esto se corroboró con los datos que presentamos a continuación.

Cuando se les consultó acerca de la importancia de la participación activa de los estudiantes para el desarrollo escolar, más del $85 \%$ de ambas instituciones coincidieron en que era importante. Sin embargo, cuándo se les preguntó si es que habían participado de alguna organización escolar, las diferencias se hicieron evidentes.

\section{TABLA 3}

¿Han participado los encuestados en organizaciones estudiantiles de su escuela?

\begin{tabular}{cccc}
\hline Institución & No & $\mathrm{Si}$ & Total \\
\hline \multirow{2}{*}{ Tradicional } & $\mathbf{6 9}$ & 17 & 86 \\
& $\mathbf{8 0 , 2 \%}$ & $19,8 \%$ & $100 \%$ \\
Alternativa & 19 & $\mathbf{1 7}$ & 36 \\
& $52,8 \%$ & $\mathbf{4 7 , 2} \%$ & $100 \%$ \\
\hline
\end{tabular}

Aunque el porcentaje de estudiantes de la institución alternativa que indicó haber participado de alguna organización escolar no fue mayoritario (47.2\%), si lo comparamos al porcentaje de estudiantes de la institución tradicional que dio la misma respuesta (19.8\%), podemos observar una mayor 138 I disposición de los primeros para participar de la escuela. 
Estos resultados, complementados con lo recogido durante el primer grupo focal, presentan una aparente mayor disposición de los estudiantes de la institución alternativa, en comparación a los de la tradicional, para comprometerse y participar de los asuntos públicos escolares. En primer lugar, presentaremos la información levantada en el grupo focal con los estudiantes de la institución tradicional y luego lo referido al grupo focal con los estudiantes de la institución alternativa.

Aunque los estudiantes de la II.EE. tradicional señalaron constantemente su descontento porque las instancias de gestión escolar no los tomaban en cuenta, sus expresiones evidenciaron poca disposición para asumir responsabilidades colectivas si esto se enfrentaba con sus intereses individuales:

...lo que vemos ahora son cosas que no nos (a los de quinto) benefician tanto, entonces si no nos benefician, los que deben buscar [alguna solución] son los de primero, segundo, tercero y cuarto. (Alex).

...nos quitan la última hora, que usualmente es hora libre que la usamos para hacer las tareas...y yo pregunto, porque me quitan mi hora libre $(\text { Karina })^{26}$.

Estas expresiones evidenciaron su resistencia para asumir responsabilidades ante su comunidad educativa e incluso frente a los estudiantes que representaban ${ }^{27}$. El que estuviesen en su último año, era la razón que daban para desentenderse de la problemática escolar, incluso cuando dichos temas, como no ser tomados en cuenta por las autoridades escolares, los había afectado por varios años ${ }^{28}$.

Los estudiantes reconocían que esta situación se debía en parte a su propio desinterés, "Un problema este año es la falta de interés de los alumnos... nadie se quiere reunir ni nada (Sandra)". Incluso ante situaciones que los afectaban directamente, como las normas escolares, los estudiantes reconocieron estar desinformados.

\footnotetext{
26 Este reclamo se sustentaba en que el momento de reuniones del consejo estudiantil era durante la última hora los días viernes, momento que los estudiantes de 5을 de secundaria consideraban una "hora libre" para ponerse al día o simplemente descansar.

27 De los 9 participantes del grupo focal, 5 de ellos eran miembros del consejo estudiantil.

28 Durante el grupo focal se repitieron varias veces, frases como "Uno intenta cambiar las cosas pero te desaniman, te cierran la puerta en la cara" (Pati).
} 
...primero tenemos que saber bien que dice el reglamento para ponerlo en práctica. Si no sabemos bien que dice el reglamento, entonces no podemos defendernos (Sandra).

Señalaron que en situaciones que podían considerar injustas y arbitrarias, los docentes usaban frases como "así lo ha establecido el colegio" y al desconocer a profundidad el reglamento no podían contradecir lo dicho. Sin embargo, cuando se les planteó que promovieran un cambio, nuevamente se mostraron reacios a comprometerse.

El reglamento si cambia, cambiaría el próximo año, y nosotros no vamos a estar, entonces no le vamos a dar mucha importancia...quinto no puede ayudar tanto (Luis).

Lo planteado corroboraría la dificultad de estos estudiantes para asumir responsabilidad ante las necesidades del colectivo, así como su poca identificación con los asuntos públicos escolares. Así mismo, lo señalado por los estudiantes mostró que se concebían como actores secundarios en la comunidad escolar. Ante la pregunta "¿Cuál es el rol que deben cumplir las autoridades escolares y docentes dentro de la escuela?", fue significativo el rol que éstos le asignaron al director como único responsable de las decisiones públicas:

Los docentes deberían dar ideas. La última palabra la debería tener el director. (Paola)

El director es el supervisor, y no se puede cambiar roles, es el que dirige (Alex).

Lo expresado nos mostró que a pesar de su expectativa de ser reconocidos como interlocutores válidos en los procesos de toma de decisión, los estudiantes legitimaban los roles establecidos y el que las autoridades escolares tuviesen el monopolio de las decisiones. Los datos mostraron que esta situación se vinculaba con el tipo de participación que los estudiantes esperaban tener. Aunque, más del $85 \%$ consideraba importante que el estudiantado participase activamente para que la escuela mejore, la mayoría prefirió canales de tipo consultivo para participar. 
TABLA 4

¿Cómo le gustaría participar a los encuestados en su escuela?

\begin{tabular}{ccccc}
\hline Institución & $\begin{array}{c}\text { Eligiendo } \\
\text { representantes }\end{array}$ & Opinando & $\begin{array}{c}\text { Opinando y } \\
\text { decidiendo }\end{array}$ & Total \\
\hline Tradicional & 14 & 33 & 34 & 81 \\
& $17,4 \%$ & $40,7 \%$ & $\mathbf{4 1 , 9 \%}$ & $100 \%$ \\
Alternativa & 0 & 7 & 26 & 33 \\
& $0,0 \%$ & $21,2 \%$ & $\mathbf{7 8 , 8 \%}$ & $100 \%$ \\
\hline
\end{tabular}

Cuando se les consultó a los estudiantes cuál consideraban que podía ser su aporte para el mejoramiento de la escuela, sus opiniones reforzaron los datos. Nuevamente, en el caso de las normas escolares, cuando se les preguntó qué podrían hacer para que las reglas que nos les gustaban cambiasen, sus respuestas evidenciaron la poca capacidad de agencia que reconocían tener.

"Nada...Nosotros quisiéramos hacer algo, pero si no nos dejan, no podemos." (Karina).

Esto se observó de manera recurrente en el grupo focal y a pesar de su descontento, los estudiantes percibían la situación escolar como inalterable, a menos que el cambio proviniese de las autoridades. Las siguientes intervenciones, aunque muestran su descontento, también indicarían que su mayor expectativa era ser escuchados "de vez en cuando", lo que evidenciaría su interiorización de un rol secundario en la institución.

Que reconozcan que no somos ningunos taraditos...quizá no vamos a ser nosotros los que decidamos las normas pero que pidan la opinión de los alumnos (Sandra).

No creo que debamos hacer las normas, porque como somos lo tomaríamos muy infantilmente, pero...que nos tomen en cuenta (Pati).

La información levantada en la II.EE. tradicional indicaría bajos niveles de compromiso de sus estudiantes ante la cotidianeidad pública escolar, pocas expectativas de éstos para asumir un rol más protagónico en los procesos de toma de decisión escolar, así como dependencia ante el accionar de las autoridades escolares. 
En el caso de la institución de propuesta alternativa, la situación encontrada fue distinta. Los datos de las tablas 2 y 3 indicaban que la mayoría de sus estudiantes aparentemente tenían mayor disposición para comprometerse y participar de los espacios públicos educativos ${ }^{29}$, que sus pares de la institución tradicional. Esta impresión se corroboró con la información levantada en el grupo focal.

Lo expresado por los estudiantes indicaría que éstos reconocían la responsabilidad que poseían como miembros de la institución educativa. Esto se evidenció, por ejemplo, a través de sus reacciones ante la salida de quien había ocupado la dirección de la institución por casi dos décadas ${ }^{30}$. En palabras de los mismos estudiantes, Ricardo era uno de los pilares de la institución y su salida generó preocupación; pero también motivación para comprometerse a que la institución continuara por el mismo camino, lo que influyó a que algunos decidieran postularse al consejo estudiantil.

Claro, estamos más comprometidos, se va [Ricardo], entonces, hay que hacer, empieza a moverte...la lista para el [consejo estudiantil] fue formada porque dijimos vamos a hacer algo (Mirna).

Nosotros mismos estamos generando los cambios... El [consejo estudiantil] se está esforzando, se está metiendo, está coordinando (Martha).

Lo recogido fue indicativo de la disposición de estos estudiantes para comprometerse con la institución y del rol que entendían que les correspondía. Éstos señalaron que al ser miembros de la comunidad escolar, les correspondía un rol importante frente a su desarrollo y aunque reconocían que las autoridades eran las llamadas a liderar la institución, consideraban que dicho liderazgo debía ser inclusivo y respetuoso de todos los actores.

Hay algunas ideas que sólo surgen del consejo estudiantil, pero se ven luego tanto con los alumnos como con los profesores, y luego se busca tomar una decisión. Lo importante es que el director y profesores no te

29 De los estudiantes encuestados de la institución alternativa, 75\% indicaron que su prioridad como estudiantes era aportar al desarrollo de la escuela, mientras que sólo $50 \%$ de la institución tradicional indicaron lo mismo. Así mismo, mientras que aproximadamente $50 \%$ de los estudiantes de la institución alternativa señaló haber integrado algún espacio público escolar, sólo el $20 \%$ de sus pares de la II.EE tradicional indicaron lo mismo. 
imponen nada, te escuchan. Puedes hacer muchas cosas que cambian el colegio, porque el director te escucha (Laura).

Si la mayoría está accediendo a algo, tienes que dejar de lado tus caprichos, pero tampoco es, si no estás de acuerdo, tirarte a un costado a ver lo que hace el resto, sino que...te tienes que involucrar y responsabilizarte (María).

Los comentarios evidenciaron su sentido de responsabilidad ante el espacio escolar y su reconocimiento de que debían participar a través de propuestas fundamentadas, que apuntasen a la mejora de la vida institucional y no que respondiesen a "cualquier berrinchito" 31 . Esto se vincularía con la expectativa que los estudiantes tenían de participar activamente en los espacios públicos escolares.

La tabla 4 mostró que el 78\% de los encuestados eligieron canales de participación que les permitiese involucrarse en los procesos de toma de decisión escolares. Las expresiones recogidas evidenciaron, de manera complementaria, su valoración por participar, no sólo opinando, sino tomando decisiones sobre su comunidad.

A través de la participación cada uno puede dar su punto de vista, para que tu opinión se analice y se complemente con la del resto... y se tomen mejores decisiones (Mirna).

Lo importante es siempre llegar a acuerdos, que favorezcan tanto a alumnos como a profesores... a veces los profesores pueden ceder como los alumnos (Carlos).

La información evidenciaría que los estudiantes de la II.EE. alternativa se percibían como actores importantes y con capacidad de agencia dentro de su comunidad educativa, lo cual tendría un correlato ante su disposición a comprometerse y a participar de la institucionalidad escolar.

Aunque a partir de la información presentada no es posible determinar una diferencia significativa entre los estudiantes de ambas instituciones, consideramos que sí es posible aproximáramos a identificar mayor disposición en

31 "Tampoco es el extremo de entregarte cualquier berrinchito...[por lo que] se tiene que presentar algo bien organizado." (Carlos). 
un mayor porcentaje de estudiantes provenientes de la propuesta alternativa, para comprometerse y participar del espacio escolar.

\section{La influencia de las propuestas educativas}

A continuación presentaremos los hallazgos referidos a la cotidianeidad de ambas propuestas y a cómo éstas podrían haber influido en las disposiciones de sus estudiantes. Primero, desarrollaremos los hallazgos de la II.EE. alternativa y luego lo encontrado en la institución tradicional.

Los resultados presentaron indicios de que dichas diferencias podían deberse a tres aspectos: por un lado, cómo se promovía que los estudiantes se identificasen con su comunidad educativa; por otro lado, la consolidación de espacios que permitiesen a los estudiantes asumir responsabilidades frente al colectivo y la vida institucional; y finalmente, el reconocimiento de la capacidad de agencia de éstos en la vida escolar.

\section{La propuesta alternativa}

En relación a la construcción de un sentido de pertenencia, un primer elemento a destacar de la propuesta, es la institucionalización del afecto como pilar de la cotidianeidad escolar. En la conversación con el director, este señaló que se buscaba promover y consolidar relaciones horizontales y afectivas entre los actores educativos, debido a su importancia para facilitar el aprendizaje y la construcción colectiva de la comunidad escolar.

Al colegio lo definiría como un proyecto educativo, en el que se han generado sobre todo, un clima de relaciones humanas a nivel de alumnos, profesores y directivos...las cosas que se dan en término de relaciones y de conocimientos, tienen a la base un vínculo que se establece entre personas (Director).

Lo señalado se constató durante las observaciones y fue corroborado por los mismos estudiantes durante el segundo grupo focal, cuando se les consultó que era lo que más valoraban de su institución. Éstos destacaron el trato afectivo y las relaciones que tenían la oportunidad de establecer con sus 
pares y con los adultos, a los que en muchos casos consideraban referentes y amigos.

Los estudiantes van llegando al colegio y a medida que entran se van acercando no sólo a sus compañeros, sino a compañeros de otros grados y a profesores de distintos años, a los que saludan y con los que conversan, bromean y en muchos casos juegan. Se ven incluso niñas y niños de los primeros grados abrazar y jugar con los estudiantes de años mayores. (Observación, primer día).

La relación con los profesores, el trato horizontal, como amigos. Por ejemplo con Ricardo, lo llamábamos por su nombre...el profesor está a la misma altura que el director, que los alumnos y del que limpia, porque todos somos iguales (Cristian).

Adicionalmente, otro aspecto que aportaba a generar un sentido de pertenencia en los estudiantes era la institucionalización de espacios y actividades que buscaban generar que éstos se identificasen con la comunidad educativa. Uno de estos espacios eran las "asambleas", en que se reunían los miembros de la comunidad para trasmitir información sobre temas institucionales, para organizar actividades y para definir acuerdos sobre la convivencia escolar. Institucionalmente se desarrollaban tres tipos de asambleas ${ }^{32}$, pero profundizaremos en dos de ellas para evidenciar su importancia.

Las asambleas de ciclo ${ }^{33}$, se programaban en cada bimestre dependiendo de las necesidades de los grupos. Por un lado, el espacio servía para que los grados pudiesen compartir trabajos o discusiones desarrolladas en las aulas y por otro lado, se abordaban temas que fuesen importantes para la convivencia ${ }^{34}$.

Un ejemplo de esto se observó en una asamblea de cuarto ciclo ${ }^{35}$, en la que se abordó el incumplimiento de tareas. La asamblea estuvo marcada por la reflexión sobre el tema, propiciada por los tutores, y por la discusión sobre qué debía hacerse al respecto. Si bien, los tutores asumieron el rol de monitorear la discusión, los principales involucrados del debate fueron los estudiantes.

\footnotetext{
32 Las generales, las de ciclo y las de aula.

33 Cada ciclo académico está compuesto por tres grados, desde inicial a 50 de secundaria.

34 Temas sobre los cuáles fuese necesario generar acuerdos colectivos o resolver conflictos específicos. 
Fue llamativa la preocupación de muchos estudiantes principalmente porque el incumplimiento de tareas se proyectase hacia los grados menores como reflejo de "mediocridad" e hicieron hincapié en que al ser los mayores debían asumir su responsabilidad como modelos de los más pequeños y ser más coherentes con la propuesta educativa. Al final de la asamblea se llegó a la conclusión de que existía la necesidad de revertir dicha situación y se acordó legitimar que el cuerpo docente fuese más exigente al respecto. Así, la asamblea sirvió para debatir el problema, para buscarle una solución colectiva y para que los estudiantes ejercieran control social en relación al mantenimiento de los acuerdos y principios escolares.

Por su parte, las asambleas de aula tenían una programación semanal de tres horas académicas en la mayoría de grados y eran consideradas como fundamentales en la cotidianeidad institucional:

Las asambleas de aula tienen un horario regular...el manejo de la asamblea, y el planteamiento de los temas es un asunto que es compartido por el tutor y los estudiantes... (Director).

El colegio te da la posibilidad de saber cuáles son tus deberes y derechos y los ejercitas, en las asambleas y si no estás de acuerdo con algo, puedes expresar tu desacuerdo. Claro, aunque seamos mayoría no podemos decir que queremos tres horas de recreo. Lo importante es siempre llegar a acuerdos que favorezcan tanto a alumnos como a profesores, al colegio pues (Carlos).

En las asambleas de aula el salón puede decir que no está de acuerdo con algo y eso el profesor lo puede trasmitir en la asamblea de profesores $o$ del colegio. (Carla).

Las asambleas eran valoradas porque se consideraba que a partir del encuentro constante entre los actores escolares se fortalecían los vínculos de afecto, respeto y reconocimiento. Así mismo, destacaron que a través del intercambio y debate sobre asuntos del aula y la escuela, los estudiantes se involucraban en los asuntos públicos escolares y asumían dichos temas como de interés colectivo ${ }^{36}$.

36 Otros dos espacios institucionales y que fueron muy reconocidos y valorados por los estudiantes fueron las "Olimpiadas" y "Campamentos". Ambas actividades, con dinámicas y en momentos distintos, apuntan a que los estudiantes vayan fortaleciendo las relaciones y los vínculos con sus 
En relación al segundo aspecto mencionado, se evidenció que desde la cotidianeidad de la institución alternativa se promovía que los estudiantes asumiesen un rol activo en la organización, cuidado y desarrollo de la convivencia cotidiana. Además del espacio de asamblea, antes explicado, otro recurso utilizado permanentemente eran las llamadas "responsabilidades".

Hay un período de 15 minutos [por las mañanas] de responsabilidades, corregir tareas, barrer el salón, ver quien ha faltado, son nuestras responsabilidades (Mariana).

Los chicos todos los días, cada día, tienen que hacerse responsable de alguna actividad dentro de su salón, frente al grupo del que son parte... (Director).

A través de dicho recurso cada estudiante asumía una tarea que aportase al grupo o que aportase al colegio en general. Este recurso era utilizado desde inicial hasta quinto de secundaria y se promovía que los trabajos fuesen rotativos ${ }^{37}$.

Los estudiantes cumplen sus responsabilidades y el tutor se acerca supervisando y brindando ayuda. Pegado en la pared, hay un cuadro donde se indica la responsabilidad de cada estudiante. (Observación, 5o de secundaria).

El salón se encuentra bastante sucio y desordenado. A la hora de asamblea el tutor pide a los estudiantes que se reflexione respecto a dicho tema, explicándoles que deben ser coherentes con los principios del colegio, que ellos mismos piden que la institución promueva. (Observación, 4ㅇde secundaria).

Las observaciones nos permitieron corroborar que los estudiantes tenían el hábito de cumplir de las tareas que les correspondía y que éstas eran monitoreadas por el colectivo durante las asambleas ${ }^{38}$.

pares y con los otros estamentos que conforman la comunidad escolar. Son momentos más lúdicos a través de los cuáles se busca promover actitudes como de trabajo en equipo, organización, respeto y tolerancia a los otros y porque no, diversión.

37 La elección de estas tareas, en los casos observados, se dio a partir de la propuesta y votación de los mismos estudiantes.

38 Otro recurso aprovechado para involucrar a los estudiantes con el colectivo, eran las evaluaciones, tanto de los docentes, como de sus propios pares. Las primeras, se aplicaban dos veces al año y eran insumos importantes en la evaluación semestral y anual de los docentes. Las segundas, 
Finalmente, en relación al reconocimiento institucional de la capacidad de agencia de los estudiantes, éstos valoraban la apertura institucional para recibir sus propuestas, así como la existencia de espacios escolares que les permitiese hacerse escuchar y promover cambios.

$Y$ tienes opción a decir lo que te gusta, lo que no te gusta... y buscas un cambio....No tienes que conformarte...porque sabes que puedes cambiar las cosas (Verónica).

Entre dichos espacios institucionalizados destacaban las asambleas, antes descritas, y el consejo estudiantil. Dichas instancias eran reconocidas como recursos explícitos que buscaban canalizar la participación de los estudiantes y fomentar el desarrollo de capacidades, como el diálogo, la reflexión y la toma de decisiones colectivas.

El consejo estudiantil era una instancia que poseía legitimidad ante los estudiantes debido a que reconocían que sus propuestas eran tomadas en cuenta por las autoridades escolares. Ejemplo de esto, fue lo que el director nos contó acerca del cuestionamiento de los estudiantes ante las "formaciones" a la salida.

...y el consejo estudiantil empezó a cuestionar abiertamente [los espacios de las] salidas y decir que hay muchos otros espacios en que se [pudiesen] cumplir los acuerdos de auto control y orden... Entonces, a partir de los planteamientos del consejo...buscamos probar otro estilo de salida. (Director).

Ahora bien, a pesar del reconocimiento institucional de la instancia y de la valoración de los estudiantes, la segunda parte de la investigación, puso en evidencia que el consejo estudiantil enfrentaba limitaciones institucionales. Los mismos actores plantearon la necesidad de promover cambios con la finalidad de que el espacio fuese más eficiente para la participación estudiantil, así como en su rol formativo. Lo recogido evidenció que el consejo había recuperado presencia institucional debido más a la motivación de los estudiantes que a las condiciones institucionales que se promovían. 
Ahorita está funcionando bien, pero normalmente, los alumnos no lo [al consejo estudiantil] han tomado en cuenta...las cosas están yendo muy bien con las personas que lo dirigen (Martin).

Bueno, eso [el involucramiento en los asuntos escolares] ya mucho depende de algunos equipos de los consejos estudiantiles, en los que hay algunas personas que le imponen un ritmo particular... [este consejo] exigen reuniones con los profesores cuando quieren plantear modificaciones de ciertos acuerdos o normas...(Director).

Aunque estos comentarios corroboraron la apertura institucional para que el consejo se involucrase en los temas públicos, lo que llamó la atención fue que la fortaleza de la instancia se explicó sólo a partir de lo que los estudiantes hacían. Si bien, el consejo era autónomo sobre las decisiones que adoptaba y los temas que abordaba, lo planteado evidenció que como recurso pedagógico tenía dos vacíos institucionales: el débil acompañamiento institucional y que no existiera tiempo definido para sus reuniones y organización.

El bimestre pasado, como que flojeamos un montón; ya estábamos medias cansadas, porque era reunirnos en el recreo y en las vacaciones también venir al colegio. (Martha).

... pero también se te van las ganas de participar porque no tenemos dirección. Nos reunimos y no sabemos qué hacer (Carlos).

Hay un asesor del consejo estudiantil, pero en la práctica no es tan real... los asesores tienen múltiples responsabilidades y como que "descuidan" esta responsabilidad (Director).

Así pues, que no hubiese tiempos institucionalmente establecidos para que los estudiantes se organizaran, así como que éstos no contasen con asesoramiento adulto -como sí sucedía en el caso de las asambleas- eran aparentes barreras en el fortalecimiento de la instancia y provocaba desgaste en los estudiantes.

El análisis de algunos aspectos de la propuesta alternativa pone en evidencia que en un espacio de formación como el escolar, además del reconocimiento y valoración de los estudiantes, es importante reconocer la necesidad de que institucionalmente se apoye y acompañe la consolidación de espacios de participación, para que éstos se conviertan en experiencias de aprendizaje. 


\section{La propuesta tradicional}

Un primer elemento a destacar, es que cuando se les preguntó a los estudiantes de la II.EE. tradicional por el "Equipo de Gestión"39, éstos se refirieron a dicha instancia como:

\section{"La Mafia" (varios estudiantes a la vez)." \\ "El monstruo de cuatro cabezas" (Gina).}

La poca valoración que los estudiantes evidenciaron antes sus autoridades, se articulaba a la percepción de éstos sobre la inexistente preocupación de las autoridades por fortalecer lazos de confraternidad entre los distintos actores escolares. Un ejemplo planteado fue lo sucedido el día del maestro, en que las autoridades no permitieron que se organizase ninguna celebración.

"No hay vida escolar, porque no te dejan" (Karina).

"Una razón de desinterés con las cosas del colegio, es porque la mayoría de gente está en descontento con...el equipo de gestión, que en verdad hacen difícil que podamos querer al colegio...uno está con ánimo y puaj te lo bajan." (Pati).

Ahora bien, a diferencia de lo planteado, el director señaló que institucionalmente había un interés por promover un espacio escolar en que los estudiantes se sintiesen contentos, tanto en relación a sus logros académicos, como en relación al vínculo que pudiesen establecer con los docentes. Sin embargo, cuando profundizó en los cambios que se estaban promoviendo sólo se hizo mención a temas académicos ${ }^{40}$.

Los cambios, en general, son de tipo académico, ampliando la oferta académica. Sería desarrollar a los alumnos a su potencial máximo... no todos van a tener los mismos logros, pero que tengan una base sólida (Director).

39 Instancia en la que participaban distintas autoridades para la dirección institucional.

40 Hay que señalar que el director del colegio, al momento de la investigación, tenía sólo un par de años de gestión, por lo que quizá dicha propuesta era pensando a futuro. 
La percepción de los estudiantes acerca de un espacio escolar distante se corroboró además, cuando éstos señalaron que promoverían valores que los ayudasen a formarse con "carácter", que les permitiera enfrentarse al "resto" y soportar "ataques" de otros. Según lo planteado, estos ataques provenían en muchos casos de los mismos docentes, quienes, con algunas excepciones, poseían actitudes negativas hacia los estudiantes, como se pudo observar.

La profesora dirigiéndose al salón les dice "mi labor es enseñarles, no cultivarlos", haciendo referencia al desarrollo de los contenidos. (Observación de 5 to $B)$.

Docente pone un video y a la mitad lo para y hace preguntas. Uno de los alumnos responde algo, y el docente le dice: "no he puesto el video para que duermas". El alumno contesta, "pero profe, he estado escuchando, pregúnteme algo"; "aja, por supuesto estas escuchando", responde el docente y marca negativo en el registro (Observación de 4to A).

Aunque se dio en el menor de los casos, se pudo observar docentes que mostraron en sus clases un trato amable y respetuoso frente a los estudiantes, sin embargo, el que no fuese una práctica recurrente indicaría de que dicha actitud respondía más a la disposición de algunos docentes, que a la propuesta institucional.

Otro elemento que se evidenció y que aparentemente limitaba las posibilidades de fortalecer el sentido de pertenencia de los estudiantes, fue que el único espacio de encuentro entre los miembros de la comunidad escolar, llamado "asamblea"41, estaba marcado por relaciones verticales y distantes. Durante las asambleas los estudiantes se sentaban en las bancas que les correspondía según los grados y eran dirigidas desde el estrado por el director o en su defecto por el coordinador del nivel ${ }^{42}$. A continuación presentamos un extracto de la descripción de una de las asambleas observadas ${ }^{43}$ :

Cuando entra el coordinador de secundaria, un [brigadier] dice a viva voz "todos de pie" y todos los alumnos se quedan asi hasta que éste sube

41 Espacio que se reproducía diariamente entre las 8.00 y las 9.00 de la mañana en el auditorio de la institución, tanto para primaria como para secundaria.

42 Aunque la institución era laica, el objetivo principal de las asambleas era la lectura y reflexión acerca de algún pasaje bíblico, lo que se consideraba beneficioso para los estudiantes.

43 Esta dinámica se repitió los cuatro días de observación. 
al estrado; el coordinador saluda y luego que los estudiantes responden el saludo se sientan. El coordinador da el sermón parado en el estrado. Hay un [brigadier] por sección cuidando que todos estén en silencio. Los docentes también vigilan...se paran a los lados de las bancas mirando a los estudiantes y haciéndolos callar cuando es necesario. (Observación del colegio tradicional).

Aunque la asamblea era una tradición escolar y el único momento cotidiano de encuentro, los estudiantes mostraron su desencanto ante su modus operandi.

Nosotros podríamos conseguir el $90 \%$ de las firmas de las personas que ya no quieren que haya asamblea...pero el colegio dice 'no pues', a pesar de que sea una mayoría. (Sandra).

Lo que pasa es que la asamblea es una tradición...pero las tradiciones tienen que irse acoplando con la modernidad. Lo que debería hacerse es cambiar la forma en que se hacen las asambleas. (Karina).

Estos comentarios demuestran el deseo que tenían los estudiantes por transformar el espacio y la frustración que sentían al no encontrar los medios para lograr algún cambio. Esto último se vincula con el reconocimiento y valoración de la institución escolar hacia las propuestas y el rol de los estudiantes en la vida pública institucional. Cuando se le preguntó al director sobre qué aspectos de la vida escolar podían influir los estudiantes, éste señaló:

Teóricamente sobre todo, pero, como por ejemplo que encuentran a veces las asambleas un poco aburrido, que a lo mejor es cierto para ellos, puede ser, pero eso ha sido algo tradicional y fundamental en la vida del colegio desde el día que empezó y eso no va a cambiar. (Director).

Su comentario evidenció que había temas de la cotidianeidad sobre los que los estudiantes no tenían opinión, incluso cuando éstos los tocaban directamente. Los estudiantes eran conscientes de esto y evidenciaron su resentimiento ante dicha situación.

Yo en lo personal hablé con [el supervisor de secundaria], de buscar que las asambleas sean más atrayentes. El problema es que se habló en el 
consejo [estudiantil], con [el director académico] estando ahí y dijo 'caso cerrado hablemos de otra cosa'. O sea, no dejaron que eso pasara a mayores (Sandra).

Este relató indicaría que los estudiantes no eran considerados interlocutores válidos con los que se pudiese debatir sobre aspectos centrales o tradicionales de la propuesta educativa. Esta situación se conectaba con la percepción de las autoridades acerca del rol de los estudiantes en la institución, como lo planteó el director cuando se le preguntó sobre cómo se promovía la participación de los estudiantes:

Mayormente en los salones de clase. Básicamente el profesor pone en instancias en la cual el alumno tiene que investigar por sí mismo, tiene que exponer por sí mismo, pero más que tener la información tiene que saber utilizarla. Y eso desarrolla en el alumno la posibilidad poco a poco de elegir, buscar, tomar decisiones y exponer en la forma en la cual él crea conveniente e interesante. (Director).

Las actividades mencionadas estaban orientadas a que los estudiantes se hicieran responsables de su desarrollo académico, con el propósito de desarrollar su autonomía y disciplina frente al trabajo. Esto, aunque valioso para el desarrollo personal de los estudiantes, se enfocaba en su dimensión individual, mientras que se dejaba de lado el fortalecimiento del vínculo de éstos con la cotidianeidad y los temas públicos escolares. En las observaciones se evidenció que incluso en cuestiones básicas del grupo, como la limpieza de los ambientes que éstos usaban, los estudiantes no asumían ninguna responsabilidad.

Lo descrito indicaría que la propuesta institucional priorizaba la dimensión académica para promover la participación y el desarrollo del sentido de responsabilidad de los estudiantes. Esto se debía en parte al poco reconocimiento de los estudiantes como posibles aportes en los asuntos públicos y era producto también de las pocas posibilidades de representación que éstos tenían, incluso a través de los "brigadieres" ${ }^{44}$ y el consejo estudiantil.

44 En la institución tradicional a los estudiantes que asumían esta función se les llamaba por otra denominación, pero decidimos usar el término brigadier para que no poner en evidencia a la institución. 
Los brigadieres ${ }^{45}$, eran estudiantes de quinto de secundaria, quienes eran elegidos por las autoridades escolares en base al aprovechamiento y comportamiento que hubiesen demostrado en sus años de estudio. Su función principal era apoyar con la manutención de la disciplina, pero el director planteó que en el último año se les había intentado dar el rol de vínculo entre los estudiantes y las instancias de gestión. Sin embargo, sus expresiones evidenciaron que los estudiantes no eran muy valorados en dicho rol debido a sus "juicios inmaduros". Esto y que los estudiantes percibieran a los brigadieres como "ayudantes" del equipo de gestión y no como sus representantes, incidía en su insatisfacción.

Dicha sensación se reforzó durante el grupo focal cuando los estudiantes señalaron unánimemente que lo que menos les gustaba del espacio escolar eran sus limitadas posibilidades para participar, incluso a través del mismo consejo estudiantil. Los estudiantes plantearon su disconformidad ante la poca relevancia que se le daba a dicha instancia y a las restricciones institucionales que se le ponía.

El colegio sería mucho mejor si dejara participar mucho más, pero creo que en el [consejo estudiantil] no se están pudiendo hacer las cosas (Gina).

Supuestamente discutimos sobre cosas importantes, pero discutimos sobre el papel higiénico del baño, cosas como esa sin importancia (Alex).

No se toca nada importante, por ejemplo sé que no van a botar a los cinco profesores que quiero que boten....no van a hacer nada importante para el cuerpo estudiantil (Karina).

Las citas muestran que los mismos estudiantes identificaban algunos aspectos que limitaban el funcionamiento del consejo, como el que se discutiesen temas de poca relevancia para sus intereses. Esto lo corroboró el director cuando planteó que se buscaba la opinión de los estudiantes en temas como el diseño de la sudadera que usarían el siguiente año. Aunque los estudiantes pueden valorar que se les consulte acerca de una prenda que

45 "[Los brigadieres] ejercen un rol en la disciplina, tienen deberes en los patios durante los recreos y refrigerios, tienen deberes...y deberían ser un lazo entre los alumnos y nosotros con situaciones problemáticas. Por supuesto todavía sus juicios son inmaduros, pero producen sugerencias interesantes a veces" (Director). 
usarán, esto pierde total relevancia cuando sólo son consultados sobre temas que no tienen mayor influencia en la cotidianeidad institucional. Según los estudiantes ( 5 de los que participaron del grupo focal pertenecían al consejo), la discusión de temas sin relevancia, como si "se pone jabón y tachos de basura en los baños", les generaba la sensación de perder su tiempo al participar de las sesiones.

Otro aspecto que los estudiantes plantearon que limitaba su participación en el consejo estudiantil y que incidía en que lo concibiesen como inútil, fueron las restricciones para discutir temas o plantear propuestas. Las iniciativas del consejo pasaban por la evaluación del Equipo de Gestión, que tenía la última palabra para aprobar las propuestas; sin embargo, los mismos estudiantes señalaron que "nos mandan a volar a cada rato".

...y el segundo problema, es que no te hacen caso. Por ejemplo propusimos poner barras como en los bancos para ordenar el acceso al kiosko, se presentó un proyecto y no se ha hecho nada. (Alex).

Yo creo que el [consejo estudiantil] no sirve porque, yo me mate haciendo una propuesta [a la que se refiere Alex], y todavía no veo nada (Isabel).

El escenario descrito evidencia, por un lado, que se dificultaba la construcción de vínculos entre las autoridades escolares y los estudiantes y por otro lado, que se restringía su participación en los asuntos públicos escolares, lo que generaba desinterés y aislamiento de los estudiantes hacia sus asuntos privados.

\section{A modo de conclusión}

Los resultados muestran que existían diferencias entre las disposiciones de los estudiantes de ambas instituciones ante las dos cualidades democráticas analizadas. Aunque no es posible señalar que dichas diferencias son resultado directo de las propuestas educativas, sí consideramos que la información da cuenta de cómo las cotidianeidades escolares influirían en el desarrollo de actitudes y disposiciones diferenciadas entre sus estudiantes.

Los hallazgos muestran, por un lado, que la mayoría de estudiantes de la II.EE. alternativa mostraban disposición para comprometerse y participar del espacio público escolar y se reconocían como actores importantes de la cotidia- 
neidad escolar. Por otro lado, se evidencia que los estudiantes de la institución tradicional, aunque señalaban que el desarrollo escolar dependía también de ellas y ellos, en lo concreto mostraron mayor retraimiento hacia sus espacios privados y se concebían más como actores secundarios y dependientes de las autoridades.

Si concebimos que la democracia se sostiene sólo a través del fortalecimiento de una cultura democrática, un aspecto que tendría que asegurarse, como se refirió al inicio del artículo, es la necesidad de que los sujetos se apropien de la categoría de ciudadanos. Ésta, entre otras dimensiones, se caracteriza por el sentido de pertenencia de los sujetos y la disposición para involucrarse de los asuntos públicos. Partiendo de estas ideas, los resultados indicarían que mientras una propuesta educativa aportaba de alguna manera al desarrollo de dichas actitudes y disposiciones, la cotidianeidad de la otra propuesta las limitaba.

Las diferencias halladas podrían explicarse a modo de aproximación a partir de los tres aspectos analizados: el desarrollo de un sentido de pertenencia de los estudiantes ante el colectivo escolar, la institucionalización de espacios para que éstos asuman responsabilidades con la comunidad y su reconocimiento institucional como interlocutores válidos en los temas públicos.

Durkheim (1997), en sus reflexiones sobre la educación moral, señala que:

Para que el hombre sea un ser moral ... es necesario que se sienta solidario con una sociedad, por humilde que sea ... la primera tarea de la educación moral es relacionar al niño con la sociedad que lo rodea inmediatamente . . . No existe moral sino hay un sentimiento e identificación con un colectivo y sin esto no hay compromiso. (p. 94 y 97)

En relación al desarrollo del sentido de pertenencia, encontramos en la II.EE. alternativa que el reconocimiento y la valoración institucional de los estudiantes, así como la existencia de espacios cotidianos para el intercambio entre los diferentes actores ${ }^{46}$, eran importantes para promover que éstos se identificaran con el espacio escolar. Dicha propuesta promovía explícitamente un ambiente afectivo y de reconocimiento hacia los estudiantes, así como

46 No sólo en cuestiones académicas o formales, sino también a partir de relaciones lúdicas y de reconocimiento 
recursos que provocaran que éstos desarrollaran mayor responsabilidad ante el espacio escolar.

Esto generaba el desarrollo de relaciones y vínculos comunitarios. Que los estudiantes se identificasen con su comunidad escolar y que reconocieran al resto de actores como miembros del mismo colectivo, incidía en que éstos asumieran el espacio escolar como propio y por lo tanto, que reconocieran la importancia de comprometerse con su desarrollo.

Por el contrario, el tipo de relaciones distantes que se reproducían en la II.EE. tradicional y el que no se promovieran la conformación de una identidad colectiva, incidía en que sus estudiantes no se sintiesen reconocidos y por lo tanto, percibiesen los temas públicos como ajenos. Un ejemplo es que cuando se les consultó de quien creían que era la responsabilidad que se promovieran un mejor ambiente escolar, señalaron que dependía del "alma mater".

Al caracterizar la propuesta educativa tradicional en nuestro país se evidenció que una de sus características era la anonimación de los estudiantes, la cual incidía directamente en que éstos viesen limitadas sus posibilidades para fortalecer su autonomía. Lo desarrollado plantearía, que dicha anonimación incidiría también en debilitar el sentido de pertenencia y responsabilidad de los estudiantes hacia su entorno escolar.

Otro aspecto que se desprende de lo desarrollado es que el rol que se les asigna institucionalmente a los estudiantes, así como la manera en que éstos se perciben, influiría en su motivación para comprometerse y participar de los temas institucionales. Eso demostró ser una diferencia importante entre ambas propuestas educativas. En la II.EE. alternativa se reconocía a los estudiantes como actores con capacidad de influencia, percepción que los mismos estudiantes incorporaron y reclamaban. En la II.EE. tradicional, sin embargo, a los estudiantes se les asignaba un rol secundario y se limitaba sus posibilidades de participación al espacio del aula.

Dicha situación, aunque corrobora una situación de anonimación de los estudiantes de la institución tradicional, coincide también con la segunda característica desarrollada en las aproximaciones conceptuales, que indicaba que se restringe las posibilidades de participar de los estudiantes a cuestiones académicas. Esto, como reflejan los resultados, no sólo provocaba frustración en dicho estamento, sino además, incidía en su desinterés ante cuestiones públicas. Es importante señalar que si bien lograr que los estudiantes sean responsables ante su desarrollo académico es valioso, desde una perspectiva de formación democrática se hace necesario que dicho 
sentido de responsabilidad se proyecte hacia la búsqueda del desarrollo colectivo.

Ahora bien, el compromiso hacia el colectivo y la disposición a participar, surge a partir de las posibilidades que los sujetos posean para involucrarse en espacios de responsabilidad pública. Los resultados presentados muestran que institucional y cotidianamente, esta dimensión se abordaba de manera distinta en ambas instituciones.

En la II.EE. alternativa se promovía que los estudiantes asumiesen un rol activo en la convivencia escolar, a través de espacios institucionalizados, mientras que en la II.EE. tradicional los estudiantes tenían pocas posibilidades de asumir responsabilidades y de participar en la vida pública escolar. La disposición a la participación no es algo que se forme de la noche a la mañana y en tal sentido, un elemento importante de la propuesta alternativa fue la institucionalización de recursos pedagógicos- como las asambleas y responsabilidades- que contaban con un tiempo en el horario escolar, con acompañamiento adulto constante y con reconocimiento institucional sobre sus posibilidades de influencia.

Así también, lo desarrollado mostró que si estos espacios no son acompañados o reconocidos, podrían darse efectos negativos como sucedía con ambos consejos estudiantiles. En el caso de la II.EE. alternativa, el que se haya dejado que el consejo funcionase de manera "autónoma", sin acompañamiento permanentemente, incidía a que dicha instancia no fuese sostenible y dependiera exclusivamente de la motivación de sus integrantes.

En el caso de la II.EE. tradicional, aunque existían figuras de representación estudiantil sus posibilidades para involucrarse se veían seriamente limitadas por la misma institución. Por un lado, los estudiantes no elegían libremente a sus representantes, por otro lado, no tenía las facultades para involucrarse en temas relevantes y finalmente, sus propuestas se veían fuertemente restringidas según el criterio de las autoridades.

Sin embargo, y a pesar de sus propias debilidades, la propuesta alternativa demostró en su cotidianeidad mayores espacios y recursos que la tradicional, para promover la participación de sus estudiantes. Partiendo de la escala de desarrollo participativo planteado por Carbajo y Serna (1999) ${ }^{47}$, se hace evidente que mientras que los estudiantes de la II.EE. alternativa lograban alcanzar el nivel mínimo de "organización" y el máximo de "decisión", los de 
la institución tradicional alcanzaban, de vez en cuando, sólo el segundo nivel de colaboración.

Así pues, lo desarrollado nos lleva a reforzar, como se planteó al principio, la importancia de fortalecer en los sujetos, más allá del estatus legal, su sentido de pertenencia y responsabilidad hacia su comunidad como aspectos fundamentales en la consolidación de una cultura democrática. Esto facilitaría que los sujetos se sientan responsables de la construcción de un "nosotros" y que asuman un compromiso activo en el logro del bien colectivo (Mouffe, 1999).

\section{Reflexiones finales: el rol de la escuela en el proceso de democratización de la sociedad peruana}

Bourdieu (1997) señala que para consolidar estructuras de orden y convivencia social, el Estado aprovecha fuentes de producción simbólica- entre las que destaca a la escuela- para la constitución de una identidad común reconocida e interiorizada por los sujetos. Así pues, el aprovechamiento de la institución educativa para legitimar las estructuras de convivencia democrática, permitiría que la democracia no se promueva sólo desde la legalidad, sino que su reproducción se sustente en la incorporación de los sujetos de una cultura democrática.

Lo desarrollado en el artículo nos aproxima a comprender cómo la II.EE., a través de su organización, estructuras de poder y de las relaciones que se reproducen cotidianamente influye en el desarrollo de disposiciones y actitudes más, o menos, democráticas. Sin llegar a extremos, los resultados indicarían que la propuesta institucional alternativa aportaba a que sus estudiantes desarrollasen mayor disposición hacia la democracia, en comparación a la propuesta tradicional ${ }^{48}$.

Consideramos que lo presentado aporta, en primer lugar, en evidenciar que las II.EE., independientemente de si son públicas o privadas o del estrato social en el que estén inscritas, dejadas a su "libre albedrío" serían espacios sociales que reproducirían propuestas educativas "tradicionales" e incidirían en el desarrollo de disposiciones anti democráticas.

48 Ahora bien, como se mencionó, no es posible referirnos a propuestas institucionales "puras" -tradicionales o alternativas- ya que los resultados evidenciaron en la segunda también se reproducían algunos rasgos tradicionales por falta de claridad institucional. 
En segundo lugar, lo presentado evidencia que si queremos que las II.EE. aporten significativamente en la formación de ciudadanos y en la consolidación de una cultura democrática, es indispensable que la propuesta educativa reproduzca cotidianamente valores, prácticas, formas de organización, estructuras de poder y relaciones democráticas. En tal sentido, será importante que se incorporen recursos pedagógicos, transversales a la cotidianeidad y de aplicación constante, que explícitamente busquen desarrollar actitudes democráticas en sus estudiantes.

En tercer lugar, los resultados muestran que si seguimos esperando que "naturalmente" nuestra cotidianeidad social se apropie de valores y prácticas democráticas, sólo como producto de cambios en estructuras formales o de leyes, lo que seguirá sucediendo (como cada uno de nosotros experimenta día a día) será la reproducción de una cotidianeidad social y política que limita considerablemente las posibilidades de consolidar la democracia en el Perú.

Ahora bien, reconocemos que en el análisis de la educación como medio para fortalecer una cultura democrática, quedan muchas dimensiones por analizar, como si los docentes actualmente están realmente preparados para asumir el reto de formar ciudadanos democráticos y esperamos que lo presentado permita generar reflexión al respecto.

Aunque en los últimos años se han promovido iniciativas para la construcción de un proyecto nacional democrático ${ }^{49}$, un gran vacío de éstas es que no se reconoce el rol que las II.EE. deberían asumir. Si apuntamos a la consolidación de la democracia, debemos reconocer que las instituciones educativas son espacios privilegiados en la formación de ciudadanos que reproduzcan cotidianamente dichos valores y prácticas. La democracia sólo puede ser aprendida a través de la experiencia cotidiana y por tal razón, la institución escolar debería ofrecerles a los niños, niñas y adolescentes la posibilidad de crecer en un espacio que "sea congruente con los imperativos sociales y políticos de la democracia" (Giroux, 1993, p. 132).

49 Como el Acuerdo Nacional o el Proyecto Educativo Nacional (Consejo Nacional de Educación, 2006). 


\section{Referencias Bibliográficas}

Ames, P. (1999). El poder en el aula: Un estudio en escuelas rurales andinas. En M. Tanaka (Ed.) El poder visto desde abajo: democracia, educación y ciudadanía en espacios locales, (pp. 267-330). Lima: IEP.

Apple, M. (1996) El conocimiento oficial. La educación democrática en una era conservadora. Buenos Aires: Paidós Ibérica.

Benavides, M., V. Villarán \& S. Cueto (1999). Socialización escolar y educación en valores democráticos: El caso de las escuelas alternativas. En: Panfichi y Valcárcel. Juventud: sociedad y cultura (pp. 133-170). Lima: Red para el Desarrollo de las Ciencias Sociales en el Perú.

Benavot, A. (1996). Education and Political Democratization: Cross-National and Logitudinal Findings. Comparative Education Review, 40 (4), 377-403.

Bobbio, N. (1989). Liberalismo y Democracia. México D.F: Fondo de Cultura Económica.

Bourdieu, P. (1997). Razones Prácticas. Sobre la Teoría de la Acción. Barcelona: Anagrama.

Carbajo, J. L. \& Serna, A. (1999). Municipio escolar: Pistas para el ejercicio ciudadano en la escuela. Lima: Tarea Asociación de publicaciones educativas.

Consejo Nacional de Educación. (2006). Proyecto Educativo Nacional al 2021: la educación que queremos para el Perú. Perú: CNE.

Cotler, J. (2001). Navegar contra el viento: las elecciones y la democracia en el Perú. En M. Vargas Llosa (Ed.), Cómo Fujimori jodió al Perú (pp. 191-207). Lima: Milla Batres.

Durkheim, E. (1997). La educación Moral. Buenos Aires: Lozada.

Gamio, G. (1999). Explorando la Democracia. En V. Santuc; G. Gamio \& F. Ghamberlain (Eds.), Democracia, Sociedad Civil y Solidaridad (pp. 145-162). Lima: Escuela Superior Antonio Ruiz de Montoya, Instituto de Ética y Desarrollo.

Giddens, A. (2002). Sociología. Madrid: Alianza Editorial.

Giroux, H. (1993). La escuela y la lucha por la ciudadanía. México: Siglo Veintiuno.

Guttman, A. (2001). La educación democrática: Una teoría política de la educación. Buenos Aires: Paidós Ibérica.

Iguiñiz, M. \& Dueñas, C. (1998). Dos miradas a la gestión de la escuela pública. Lima: TAREA. 
Kalinowski D.; A. Ruiz \& C. Dueñas (1996). Escuelas que construyen democracia: Siete experiencias innovadoras en el Perú. Lima: CIDE y EDUCALTER.

León, E. \& Staeheli, M. A. (2001). Cultura Escolar y Ciudadanía: Investigaciones etnográficas en Ayacucho y Lima. Lima: TAREA.

Mead, G. (1972). Espíritu, persona y sociedad. Desde el punto de vista del conductismo social. Buenos Aires: Paidós.

Moragues, M. (1996). Hacia la escuela posible. Sistematización del proyecto educativo del colegio La Casa de Cartón. Lima: Tarea Asociación de publicaciones educativas.

Mouffe, C. (1999). El retorno de lo político. Comunidad, ciudadanía, pluralismo, democracia radical. Barcelona: Paidós.

Stojnic, L. (2006). La escuela, un espacio para la democratización peruana. Un estudio comparado entre la propuesta educativa tradicional y una propuesta educativa alternativa. Tesis de Licenciatura, Pontifica Universidad Católica del Perú, Perú.

Toqueville, A. (1984). La democracia en América. Madrid: Sarpe.

Touraine, A. (1995). ¿Qué es la Democracia? México: Fondo de Cultura Económica. 